\title{
Kinetics of Nonequilibrium Gas Phase Reactions Induced by Vibrational Multiple-Photon IR Laser Excitation of Molecules
}

\author{
V. N. BAGRATASHVILI and M. V. KUZMIN \\ Laser Chemistry Laboratory, Research Centre for Laser Technology, Acad. Sci. USSR, \\ Troitzk, Moscow District, 142092 USSR
}

\section{S. LETOKHOV}

Institute of Spectroscopy, Acad. Sci. USSR, Troitzk Moscow District, 142092 USSR

A simple theoretical model for the qualitative description of the kinetics of the unimolecular decay in the pulsed IR laser field with strong deviation from thermal equilibrium is developed. The nonequilibrium character of the reaction is introduced by means of a special parameter of "truncation" of vibrational energy distribution function, which takes into account depletion of the distribution function due to the dissociation. The derived self-consistent system of differential equations describes the three dissociation regimes. Besides, the apparent cases of collisionless (short duration of laser pulse $\tau_{p}$ ) and equilibrium (long $\tau_{p}$ ) reactions, the intermediate collisionalnonequilibrium dissociation is revealed. The contribution of postpulse reaction has proved considerable in case of both long and short pulses. For the case of a successive dissociation the possibility of synthesis of labile products which cannot be produced at thermal initiation is established.

\section{INTRODUCTION}

At the infrared multiple-photon (IR MP) excitation of low-pressure (several Torrs) molecular gases it is possible in a very short time (fractions of microsecond) to produce sufficiently high concentrations of molecules excited to high vibrational states and free radicals, the products of their unimolecular dissociation. The interest shown in 
IR MP photochemistry ${ }^{1-7}$ is caused by the following. First, it can be realized easily since many molecules have absorption bands in the tuning range of a simple, accessible and high-power $\mathrm{CO}_{2}$ laser. Secondly, it yields quite a different composition of final products, as compared with the known methods of initiation, and enables, among other things, realizing some ways of chemical reactions which are impossible under equilibrium conditions.

The most important thing for such nonequilibrium reactions is a high rate of excitation and subsequent reactions, both primary (unimolecular dissociation) and secondary (radical recomination and other bimolecular reactions). Therefore, as the molecules are excited by a high-power short IR pulse, the reaction may take place in times shorter than the relaxation times $T_{V-T / R}$ and $T_{V-V}$. As a result, a typical IR MP-induced reaction proceeds under extremely nonstationary and nonequilibrium conditions when there is no equipartition of energy between different components of the mixture and different degrees of freedom of a single molecule. The absence of reaction rate enhancement on the cell's walls also acts a certain part since the laser excitation of molecules takes place in the space.

The nonequilibrium character of such a chemical reaction makes the standard methods of calculation of thermal chemical kinetics unapplicable. The absence of thermal equilibrium during the reaction draws nearer IR MP-induced chemistry and conventional visible-or UV-photochemistry or plasma chemistry. But an immediate transfer of the reaction description methods developed here is impossible, mainly due to quite different ways of energy deposition. In photochemistry and plasma chemistry the energy is deposited through electronic degrees of freedom. IR MP excitation brings about direct excitation of vibrational degrees of freedom, and the whole process is most commonly limited by the ground electronic state.

So the theoretical analysis of the kinetics of IR MP-induced reactions calls for the development of a special approach, one of its possible versions described in this paper. In Section 2 we shall discuss versions described in this paper. In Section 2 we shall discuss some possible ways of solving the problem and then, in Section 3, substantiate the choice of our own model. It takes up an intermediate position between the approaches known in literature and is rather simple and universal at the same time. In Section 4 the operation of main equations is illustrated on the simple limited cases (collisional and collisionless). 
In Section 5 possible reaction conditions are classified by the degree of their nonequilibrium character as (1) collisionless, (2) collisionalnonequilibrium and (3) equilibrium. In Section 6 it is shown that under collisionless and equilibrium conditions the reaction proceeds mainly after the laser pulse is over. At the same time, under collisionalnonequilibrium conditions the basic contribution is made by reactions during the pulse. In Sections 7 and 8 the reaction of successive dissociation $A B C \rightarrow^{n \hbar \omega} A B+C \rightarrow^{m \hbar \omega} A+B+C$ is studied numerically. Particular emphasis is given to the conditions under which the maximum ratio of products $[A B] /[A]$ can be obtained. It is shown that IR MP excitation makes it possible to synthesize efficiently the intermediate product $[A B]$ even in the case when it is labile and under thermal conditions dissociates faster than is formed.

Our wish was to formulate the problem widely enough. Varying over a wide range the basic parameters of reacting mixture (laser pulse duration and energy, reagent and buffer pressures, external cooling time) we were able to study the effect of each of them on the basic characteristics of the process (reaction time, ratio of different channels, laser energy utilization factor, etc.).

\section{METHODS OF MODELLING OF NONEQUILIBRIUM CHEMICAL REACTIONS}

The nonequilibrium character of the physical and chemical processes induced by MP excitation of molecules with pulsed IR laser radiation makes their theoretical analysis more difficult. Indeed, in equilibrium chemical kinetics the specification the value of one variable, temperature, determines the internal state of all the reagents. Under nonequilibrium conditions the form of vibrational distribution functions is not known and it must be determined by solving an awkward system of integro-differential equations. Most widely used is the method based in replacing the continuous distribution function with a discrete one when the discretization "grains" are usually taken to be equal to the laser quantum energy. The corresponding differentialdifference equations (energy gained master equations, EGME) have been studied in a lot of papers, their results presented in a number of reviews $^{8}$ (see, also, Refs. 1-6). This approach is applied mainly in analysis of IR MP dissociation taking place either under collisionless conditions or in the presence of a buffer gas. ${ }^{9,10}$ 
It should be noted that the discretization step equal to the laser quantum ( $\hbar \omega \approx 1000 \mathrm{~cm}^{-1}$ in the case of $\mathrm{CO}_{2}$ laser) may turn out to be too large for describing collisional deactivation if the value of average energy lost during one collision $\langle\Delta E\rangle$ is less than or of the order of $\hbar \omega$. Here it should be mentioned that the first direct measurements resulted in lower, than commonly adopted, ${ }^{11}$ values of $\langle\Delta E\rangle$ for the relaxation of highly excited $\left(E=20000-50000 \mathrm{~cm}^{-1}\right)$ molecules of azulene, ${ }^{12}$ toluene ${ }^{13}$ and substituted cycloheptatriene ${ }^{14}$ $\left(\langle\Delta E\rangle \approx 100 \mathrm{~cm}^{-1}\right.$ for the collisions with noble gases and, for example, about $500 \mathrm{~cm}^{-1}$ for the collisions with $\mathrm{SF}_{6}$ ).

For successive analysis of processes with $\langle\Delta E\rangle \leqslant \hbar \omega$ it is necessary to reduce the grains size in $\mathrm{EGME}^{10(\mathrm{~b})}$ or to return to the initial integro-differential equation. ${ }^{9(\mathrm{f}), 15} \mathrm{~A}$ serious disadvantage of EGME is a sharp increase in computer time when we try to allow for the collisions of the excited molecules between themselves, with the dissociation products of with other chemically active components. Therefore such important processes as binding and recombination of dissociation products, deactivation of excited molecules by more cold products of their decay have not been investigated with the use of EGME yet.

In this connection simpler theories applying a less detailed description of the system, with the slowest processes disregarded, have gained wide acceptance. ${ }^{16-38}$ But it is different processes that have a dominant role at different stages of reaction (for example, during a laser pulse and after it is over). So to describe the same reaction at different instants of time different theoretical models have to be applied. It is sometimes assumed, for example, that the laser pulse is short enough so that during excitation we can use simplest EGME whereas the postpulse reaction (after thermal equilibrium is established) is described by the conventional Arrhenius law. Such "hybrid" method, of course, is inevitably limited by some severe approximations. Moreover, this approach is not enough universal since there is a region of parameters (laser pulse duration and pressure) when it is necessary to take into account simultaneously the laser excitation and the collisions between excited molecules which is difficult to do within the frameworks of this hybrid approach.

As a result, the task arises to develop a more universal method of calculation of reaction kinetics that would allow for all the processes which can come about in the irradiated gas. Such a method, in 
particular, must be applicable over a wide range of reaction conditions and enable studying the change-over from collisionless to thermal dissociation at increasing gas pressure. Since, as it has been noted, the solution of EGME requires for some reaction conditions too great expenditures of computer time, it seems quite natural, at least as the first step, to get rid of attempts to construct such a theory on the basis of exact distribution function. Instead of this, we can restrict ourselves to constructing a simplified theory which will provide a correct qualitative description at all the basic reaction conditions and allow semiquantitative evaluations.

An attempt to develop such a theory was taken in work ${ }^{34(b)}$ where the theory was considerably simplified due to the assumption that the distribution function of any reacting molecule has a preset form (Boltzmann distribution) that depends only on one variable (temperature). Nonequilibrium character of the reaction was taken into account in this case by introducing different temperatures for different components of the mixture. Such an approach has enabled the most of the observed dependences to be explained. Yet we have come to the conclusion that for a correct description of some features of nonequilibrium processes (branching ratios, first of all) it is necessary to introduce another variable which allows for the truncation of distribution function caused by the rapid growth of dissociation rate as the energy increases.

\section{METHOD OF TRUNCATED DISTRIBUTION FUNCTIONS WITH DIFFERENT TEMPERATURES}

Here we are going to complicate the model with different temperatures and assume that the distribution function of any molecule is truncated at some energy $E^{D}$, as shown in Figure 1. We assume that the smooth envelope distribution function (the dashed line in Figure 1) has a specified form, and we choose it in the simplest form of Boltzmann distribution for better correspondence with equilibrium kinetics. Thus, the state of each of the molecular components is described in the present model just by three variables: average energy of equilibrium (Boltzmann) distribution function $E^{e q}$, energy of truncation of distribution function $E^{D}$ and, finally, relative concentration of molecules of the given sort $n$. On the basis of energy grained master equations (EGME) a closed system of equations can be written for these values. 


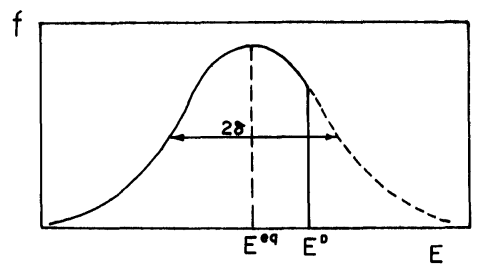

FIGURE 1 The truncated distribution function $f(E)$ (solid line) formed under IR multiple-photon excitation at rather a low pressure. With energies $E>E^{D}$ it is depleted considerably as compared to the smooth function $f^{T}(E)$ (Boltzmann distribution, for example; dashed line).

Let us consider this approach in the simplest version taking a specific reaction sequence of the polyatomic molecule $A B C$ under the action of a powerful laser IR pulse that performs MP excitation of the original molecule $A B C$ and, perhaps, the polyatomic product $A B$ of its unimolecular dissociation:

$$
\begin{aligned}
A B C+n \hbar \omega & \rightarrow A B+C \\
A B+m \hbar \omega & \rightarrow A+B
\end{aligned}
$$

This specific reaction sequence is of interest for comparison of the product composition of chemical reactions under equilibrium and nonequilibrium conditions.

To make the writing of equations more convenient, below we use the subscript " $i$ " which numbers all the components in the reacting mixture. The values $i=1,2,3, \ldots$ number different chemically active components. In our case of successive dissociation $(1 \mathrm{a}, \mathrm{b}) i=1$ corresponds to the molecule $A B C, i=2$ to $A B$ and $i=3$ to $A$. The index $i=0$ describes the inert buffer gas. Note, that nonreactive products $C$ and $B$ as well as all the translational-rotational degrees of freedom are also attributed to buffer. It is convenient to choose the concentration $n_{i}=[i] /[A B C]_{0}$, the equilibrium energy $E_{i}^{e q}$ and the average energy $\bar{E}_{i}$ as three independent variables describing each molecular component in the present model. Along with $E_{i}^{e q}$, it is convenient to introduce the temperature $T_{i}$ the molecule must have under thermal equilibrium to acquire this energy. The dependence $E_{i}^{e q}\left(T_{i}\right)$ in a harmonic approximation has the form

$$
E_{i}^{e q}=\sum_{k} \frac{h \nu_{k}}{\exp \left(h \nu_{k} / T_{i}\right)-1}
$$

where $\nu_{k}$ denotes the frequencies of normal molecular modes. 
The average energy $\bar{E}_{i}$ at given $T_{i}$ is unambiguously related to the above-introduced energy of truncation $E_{i}^{D}$. These values are present at the same time in the equations given below, and they are related as

$$
\bar{E}_{i}=\frac{\int_{0}^{E_{i}^{D}} E f_{i}(E) d E}{\int_{0}^{E_{i}^{D}} f_{i}(E) d E}
$$

where $f_{i}(E)$ is the smooth envelope function of energy disctribution. In our work it is chosen in the form of Boltzmann distribution $f_{i}(E)=$ $\rho_{i} \exp \left(-E / T_{i}\right)$, where $\rho_{i}(E)$ is the state density. We are mainly concerned with the case of a large number of vibrational degrees of freedom $s_{i} \gg 1$ when $\rho_{i}(E)$ is a rapidly increasing function. So $f_{i}(E)$ usually has a well-pronounced maximum with $E=E_{i}^{e q}$ and in specific calculations it can be approximated by Gaussian distribution with a good accuracy. Its average energy and dispersion $\delta_{i}$ are taken to be equal to the corresponding values of Boltzmann distribution

$$
\delta_{i}^{2}=c_{i} T_{i}^{2}
$$

where $c_{i}=d E_{i}^{e q} / d T_{i}$ is the vibrational heat capacity. It can be shown ${ }^{36}$ that the difference between the real distribution width and $\delta_{i}$ given by Eq. (4) is not large even under collisionless excitation if the absorption cross-section $\sigma_{i} \approx$ const.

When $f_{i}(E)$ is chosen so, the relation between $\bar{E}_{i}$ and $E_{i}^{D}$ takes on the form

$$
\bar{E}_{i}=E_{i}^{e q}-\frac{\delta_{i}}{\sqrt{2 \pi}} \exp \left\{-\frac{\left(E_{i}^{D}-E_{i}^{e q}\right)^{2}}{2 \delta_{i}^{2}}\right\} / \mathscr{P}\left(\frac{E_{i}^{D}-E_{i}^{e q}}{\delta_{i}}\right),
$$

where $\mathscr{P}(x)$ is the probability integral.

In the simplest version of the theory considered here, we shall not derive the equations for $n_{i}, E_{i}^{e q}$, and $E_{i}$ from EGME, but introduce them on a phenomenological basis using the Arrhenius-like expression for reaction rate with a correction for nonequilibrium nature of distribution:

$$
k_{i}^{U M}=A_{i} e^{-D_{i} / T_{i} \mathscr{P}}\left(\frac{E_{i}^{D}-E_{i}^{e q}-D_{i}}{\delta_{i}}\right),
$$

where $D_{1} \equiv D_{A B C}$ and $D_{2} \equiv D_{A B}$ are respectively the energies of the bonds $A B-C$ and $A-B$. 
The system of equations describing reaction (1) has the form $(i=1,2,3)$

$$
\begin{aligned}
& \frac{d n_{i}}{d t}=k_{i-1}^{U M} n_{i-1}-k_{i}^{U M} n_{i} \\
& \frac{d E_{i}^{e q}}{d t}=c_{i} \frac{d T_{i}}{d t}=\sigma_{i} I+\sum_{j=0}^{3} \nu_{i j} n_{j}\left(\frac{\bar{E}_{j} T_{j}}{E_{j}^{e q}}-T_{i}\right) \\
& \quad+\left(\alpha_{i-1}^{\prime} E_{i-1}^{D}-D_{i-1}-E_{i}^{e q}\right) \frac{n_{i-1}}{n_{i}} k_{i-1}^{U M} ; \\
& \frac{d \bar{E}_{i}}{d t}=\sigma_{i} I+\sum_{j=0}^{3} \nu_{i j} n_{j}\left(\frac{\bar{E}_{j} T_{j}}{E_{j}^{e q}}-\frac{\bar{E}_{i} T_{i}}{E_{i}^{e q}}\right) \\
&+\left(\alpha_{i-1}^{\prime} E_{i-1}^{D}-D_{i-1}-\bar{E}_{i}\right) \frac{n_{i-1}}{n_{i}} k_{i-1}^{U M}+\left(\bar{E}_{i}-E_{i}^{D}\right) k_{i}^{U M}
\end{aligned}
$$

where $I$ is the radiation intensity, $\sigma_{i}$ is the IR absorption cross-section, $c_{i}$ is the heat capacity, $\nu_{i j}$ is the relaxation constant, $\alpha_{i}^{\prime}$ is the fraction of bond energy $E_{i}^{D}$ consumed by the vibrational degrees of freedom of the product at dissociation.

The first equation (6) describes the successive dissociation according to the scheme $(1 \mathrm{a}, \mathrm{b})$. The second equation (7) describes the changes of the maximum of the distribution function due to laser excitation (the term $\sigma_{i} I$ ), collisional relaxation (including the collisions with the buffer, $j=0$, and molecules of the same type $j=i$ ) and previous component dissociation $\left(k_{i-1}^{U M}\right)$. It is assumed, however, that the dissociation of a given molecule $\left(k_{i}^{U M}\right)$ does not affect directly the shape of the envelope of the distribution function (i.e., $E_{i}^{e q}$ ) but only causes the energy of truncating $E_{i}^{D}$ to be reduced. Therefore the corresponding term (proportional to $k_{i}^{U M}$ ) is not included in the equations for $E_{i}^{e q}$ but can be found in the third equation (8) for $\bar{E}_{i}$ which is in other respects similar to (7). Only because of collisions the value $E_{i}^{e q}$ relaxes to $\bar{E}_{i}$ which is described by the second term in the right-hand side of Eq. (7). So it is unimolecular dissociation that is responsible for the difference between $\bar{E}_{i}$ and $E_{i}^{e q}$. The described behaviour becomes particularly clear if with help of Eq. (7) we rewrite Eq. (8) as

$$
\frac{d\left(E_{i}^{e q}-\bar{E}_{i}\right)}{d t}=-\gamma_{i}\left(E_{i}^{e q}-\bar{E}_{i}\right)+\left(E_{i}^{D}-\bar{E}_{i}\right) k_{i}^{U M},
$$


where

$$
\gamma_{i}=\sum_{j=0}^{3} \nu_{i j} n_{j} \frac{T_{i}}{E_{i}^{e q}}+\frac{n_{i-1}}{n_{i}} k_{i-1}^{U M}
$$

The equations describing buffer gas heating are written in a manner similar to (7) where the fraction of energy $\alpha_{i}^{\prime \prime} E_{i}^{D}$ arriving at the buffer degrees of freedom at unimolecular dissociation $\left(\alpha_{i}^{\prime}+\alpha_{i}^{\prime \prime}=1\right)$ is also allowed for.

The system presented describes all the basic processes taking place in a uniformly irradiated volume. But in this case the processes connected with the unexposed regions of gas and the cell's walls are not explicitly included. They all will lead to additional processes cooling ${ }^{39}$ due to heat conduction, diffusion, generation of sound, etc. For each of these mechanisms of relaxation it is possible to obtain a characteristic time in which it will cause a substantial deceleration of reaction. The minimum of these times is called the external cooling time $t_{\text {ext }}$. The reaction yield obtained in the text time after the laser pulse is identified as the total reaction yield. It should be noted that the real reaction time $t_{R}$ (i.e., the time in which, for example, $90 \%$ total yield is attained) can be much shorter than $t_{\text {ext }}$. This is characteristic, for example, of a reaction in the presence of a buffer gas when $t_{R}$ is determined by the collisional relaxation time.

\section{SIMPLE APPLICATIONS OF THE MODEL}

In this section we shall consider how Eqs. (6)-(8) work in some simple limiting cases (thermal and collisionless dissociation). The limits of applicability of our model will be also discussed.

Thermal dissociation. Consider the high pressure limit, where the laser pulse duration is much greater than time constants of intermolecular energy flows. In this case Eqs. (6)-(8) can be simplified considerably, and are reduced to equations of thermal chemical kinetics. Note that the dissociation rate in this case is much less than the collision rate, and Eq. (9) gives $\bar{E}_{i}=E_{i}^{e q}$, whereas from Eqs. (7) and (8) it follows that all components in the mixture should have equal temperature $T_{i}=T$. The value of $T$ can be obtained from simple energy balance (including laser heating and reaction enthalpy), the reaction rates being described by the Arrhenius law.) Now Eqs. (6)-(8) 
are reduced to customary chemical kinetics, and high pressure limit of our model appears to be well founded.

When the pressure is lowered, various nonequilibrium effects will become important. Our theory will account them, first by introducing different temperatures for different components of mixture, and second, by allowing truncation of distribution function $\left(\bar{E}_{i}<E_{i}^{e q}\right)$. Formally, present model will be valid as for as collisions have time to recover the Boltzmann shape of the distribution function, set the energies lower than the cut off $E_{i}^{D}$.

Collisionless case. If the pressure is decreased further, the vibrational distribution becomes not collision but radiation-controlled, and its form and width will not coincide with assumed Boltzmann ones. In such a case the model (6)-(8) can give only semiquantitative description of the reaction. But when constructing the model we take care to ensure that the most important feature of our approximate distribution function-its maximum $\bar{E}$-coincide with accurate one as closely as possible. It can be shown, ${ }^{36}$ that for constant absorbtion cross-section our model reproduces exact results for both the average energy $\bar{E}$ and the width of distribution even in the collisionless case.

To illustrate, that our model behaves qualitatively well in collisionless case, let us consider the dynamics of the energy of truncation $E^{D}$. This quantity is very important, because the system can be very far from equilibrium $\left(E_{i}^{D} \leqslant E_{i}^{e q}\right)$ in collisionless case. The value of $E^{D}$ is established by the competition of two processes: laser excitation and unimolecular decay. To determine on exact value of $E^{D}$ we should know the energy dependence of rate of unimolecular decay $k^{U M}(E)$, which is not incorporated to our present model. It is simulated by some nonequilibrium factor, included into Arrhenius law dissociation rate (5) which will cut the dissociation of $E^{D}$ is sufficiently small. We have carried out a multitude of numerical calculations to ensure that this approach works well enough. We have tried the broad spectrum of reaction conditions and all cases the results were reasonable.

The results of one of test calculations is shown in Figure 2. The gas was preheated by first laser pulse (not shown on figure), and then after some transient time, is irradiated once more. After the switching on of the laser $E^{D}$ is sharply increasing due to laser excitation, but to the end of the pulse $E^{D}$ is practically saturated, which is connected with competition between excitation and dissociation. At $t=1.5 \mu \mathrm{s}$ 


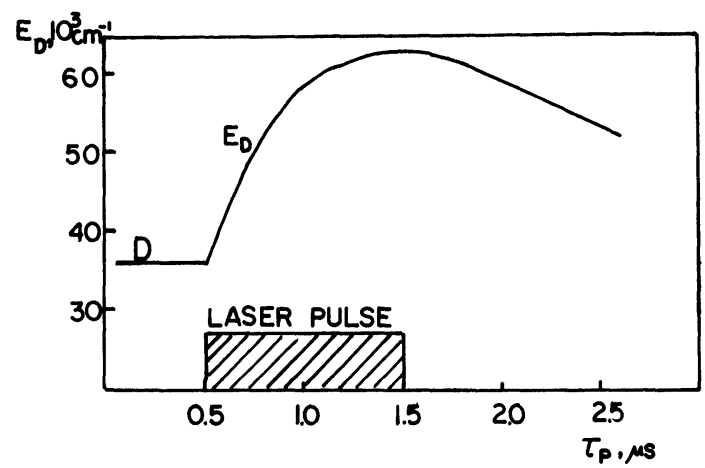

FIGURE 2 Test calculation of the reaction in collisionless case. Molecular parameters correspond to Table I (excluding pressures which are zero). Initial temperature was $700 \mathrm{~cm}^{-1}$, energy absorbed from the laser pulse was $40000 \mathrm{~cm}^{-1}$.

the pulse is switched off, and, as expected, $E^{D}$ begins gradually to decrease due to dissociation of molecules in high-energy part of the distribution function. This brings about to corresponding reduction of dissociation rate (5).

Another test also give expectable results. So we used Eqs. (6)-(8) to investigate qualitative features of nonequilibrium reactions in all regimes-from collisionless to thermal.

Proceeding to exposition of the results of our calculations, note, that numerical parameters have been chosen to correspond to experiments of Ref. 34 on the synthesis of $\left(\mathrm{CF}_{3}\right)_{3} \mathrm{CI}$ according to:

$$
\begin{aligned}
\left(\mathrm{CF}_{3}\right)_{3} \mathrm{CBr}+n \hbar \omega & \rightarrow\left(\mathrm{CF}_{3}\right)_{3} \mathrm{C} \cdot+\mathrm{Br} \\
\left(\mathrm{CF}_{3}\right)_{3} \mathrm{C} \cdot+m \hbar \omega & \rightarrow\left(\mathrm{CF}_{3}\right)_{2} \mathrm{C}:+\mathrm{CF}_{3} . \\
\left(\mathrm{CF}_{3}\right)_{3} \mathrm{C} \cdot+\mathrm{I}_{2} & \rightarrow\left(\mathrm{CF}_{3}\right)_{3} \mathrm{CI}+\mathrm{I}
\end{aligned}
$$

which is an example of successive dissociation reaction (1).

The values of basic parameters are given in Table $I$. The relaxation constants are taken in the form $\nu_{i j}=\mathscr{Z} \beta_{i j} s_{i} s_{j} /\left(s_{i}+s_{j}\right)$, where $\beta_{i j}$ is the vibrational energy exchange efficiency ( $\beta_{i j}=1$ for "strong" collisions), $\mathscr{Z}=3.75 \cdot 10^{7} \cdot P_{A B C}\left(T / 1000 \mathrm{~cm}^{-1}\right)^{1 / 2} \mathrm{~s}^{-1}$, where the pressure of $A B C$ is expressed in Torrs. 
TABLE I

The reaction conditions and model parameters used in the calculations

Molecular parameters:

Number of vibrational degrees of freedom

Dissociation energies

Preexponents

Differential cross-sections

Buffer gas heat capacity

$V-V$ exchange efficiency

$s_{A B C}=36, s_{A B}=33, s_{A}=21$

$D_{A B C}=24000 \mathrm{~cm}^{-1}, D_{A B}=22000 \mathrm{~cm}^{-1}$

$A_{A B C}=10^{14} \mathrm{~s}^{-1}, A_{A B}=10^{15} \mathrm{~s}^{-1 \text { a) }}$

$\sigma_{A B C}=\sigma_{A B}=$ const.

$c_{0}=7 / 2$

$\beta_{i j}=0.1 ; \beta_{i 0}=0.067 ; i, j=1,2,3$

\section{Reaction conditions}

(Figures 2-5)

Initial pressure of $A B C$

Buffer pressure

Time of collisionless dissociation

$P_{A B C}=0.8$ Torr

$P_{\text {buf }}=12$ Torr

$t_{c}=50 \mathrm{~ns}$

Time of setting of equilibrium conditions

External cooling time

Laser pulse duration

$t_{e q}=1.2 \mu \mathrm{s}$

$t_{\text {ext }}=100 \mu \mathrm{S}$

varied

The pulse energy was chosen according to the total reaction yield $\beta=n_{A B}$ with $t=$ $t_{\text {ext }}$ :

$$
\beta=44 \%
$$

a) The value $A_{A B}=10^{15} \mathrm{~s}^{-1}$ is used in the calculations of successive dissociation, Figure 6-9. In the series presented in Figure 2-5 it is assumed that $A_{A B}=0$.

\section{IR MP-INDUCED REACTIONS AT DIFFERENT LASER PULSE DURATIONS}

Let us consider here how the kinetics of IR MP-induced reaction and its basic characteristics change as the laser pulse duration $\tau_{p}$ is reduced, i.e., when the thermal reaction converts to a collisionless one. Such a conversion is determined by the ratio between the IR laser pulse duration $\tau_{p}$ and two characteristic times relating to collisional processes.

One time, $t_{c}$, determines the change-over from collisionless MP excitation to the conditions under which the effect of vibrational deexcitation during collisions with buffer gas particles essentially decreases the rate of MP excitation and dissociation. Let $t_{c}$ be defined as the time in which an excited molecule $A B C$ loses such a value of vibrational energy that its unimolecular dissociation rate is reduced by $e$ times. A simple estimation with the use of a semiclassical 
approximation $^{40}$ for this rate results in the following expression for $t_{c}$

$$
t_{c}=\frac{\left(\bar{E}+E_{z}\right)^{2}}{s_{A B C} D_{A B C}\langle\Delta E\rangle} \cdot t_{g k}
$$

where $t_{g k}$ is the time between the gas-kinetic collisions of the excited molecule with the buffer gas, $\langle\Delta E\rangle$ is the average energy transferred to the buffer during one collision, $s_{A B C}, E_{z}$ and $D_{A B C}$ are respectively the number of degrees of freedom, the energy of zero-point vibrations and the dissociation energy of the $A B C$ molecule.

The other, longer, time $t_{e q}$ determines the change-over to equilibrium heating of gas mixture by IR pulse due to fast redistribution of absorbed energy between all the particles and degrees of freedom in the mixture. A simple estimation gives a value for the time $t_{e q}$ during which the buffer gas is heated to the temperature equal to that of excited $A B C$ molecules with the energy $\bar{E}$

$$
t_{e q}=\frac{\left(\bar{E}+E_{z}\right)}{\langle\Delta E\rangle} \cdot \frac{n_{0} c_{b}}{s_{A B C}} \cdot t_{g k},
$$

where $n_{0} c_{b}$ is the dimensionless (divided by the Boltzmann constant) heat capacity of buffer gas per one $A B C$ molecule.

The comparison of expressions (11) and (12) shows that

$$
\frac{t_{e q}}{t_{c}} \approx n_{0} c_{b},
$$

because $\bar{E}+E_{z} \sim D$. Since $n_{0} c_{b} \sim s_{A B C} \gg 1$, this means that the relation $t_{e q} \gg t_{c}$ is always fulfilled. In our specific case the parameters of which are given in Table I we have that $t_{c}=50 \mathrm{~ns}, t_{e q}=1.2 \mu \mathrm{s}$.

Thus, according to different relations between the times $\tau_{p}, t_{c}, t_{e q}$ there are three regimes of IR MP-induced reaction of molecular gas, $\left(\mathrm{CF}_{3}\right)_{3} \mathrm{CBr}$ and $\mathrm{I}_{2}$ in our case.

$1^{\circ}$. Collisionless reaction when the relation

$$
\tau_{p} \ll t_{c}
$$

is complied with. In our case this calls for high-power laser pulses with their duration $\tau_{p} \sim 10^{-8} \mathrm{~s}$. Then reaction (10b) will proceed with cold molecules.

$2^{\circ}$. Collisional-nonequilibrium reaction when the inequalities

$$
t_{c} \leqslant \tau_{p} \leqslant t_{e q}
$$


hold true. In the case under consideration this corresponds to the most typical experiment with the IR pulse duration $\tau_{p}=10^{-7}-10^{-6} \mathrm{~s}$. In this case the MP excitation and dissociation occur in the presence of collisions, which reduce the rate of processes (10a) and (10b) to some quasistationary values, but the buffer gas temperature is yet lower than that of excited molecule.

$3^{\circ}$. Equilibrium reaction when the relation

$$
\tau_{p} \gg t_{e q}
$$

is complied with. In our case this means using comparatively long pulses with $\tau_{p} \gg 10^{-6} \mathrm{~s}$. Such a pulse is able to heat the whole gas mixture and the reaction must be equilibrium by character.

To demonstrate the main specific characteristics of different reaction regimes, Figures 3-5 present a time evolution of the basic characteristics of a single unimolecular reaction (10a) obtained by numerical integration of the system of equations described in Section 3. The influence of secondary dissociation (10b) will be described in Sections 7 and 8 below. Three different IR pulse durations $\tau_{p}$ considered here correspond to the three different reaction regimes just described. The energy density of each pulse $\Phi$ (or the absorbed energy $\bar{\varepsilon}=\sigma \Phi$ ) were chosen so that the same reaction yield $\beta=44 \%$ could be ensured on completion of the process (in the time $t_{\mathrm{ext}}=100 \mu \mathrm{s}$ ). The corresponding parameters are given in Table $I$.

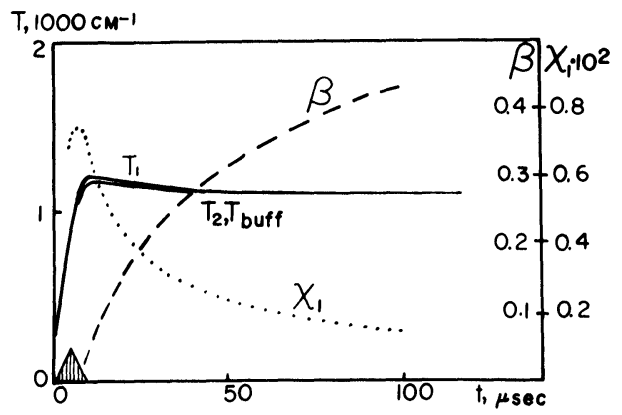

FIGURE 3 Equilibrium IR MP-induced dissociation. The time evolution of the basic characteristics of the system: the temperatures of $A B C-T_{1}, A B-T_{2}$ and the buffer$T_{\text {buf }}$; the reaction yield $\beta=[A B] /[A B C]_{0}$ and the degree of nonequilibrium $\chi_{1}$. The laser pulse is shown in the left bottom corner (shaded), its FWHM is $\tau_{p}=5 \mu \mathrm{s}$. The parameters of the model are given in Table I. 


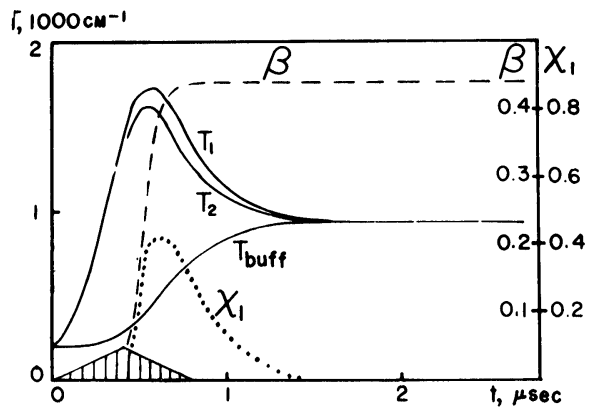

FIGURE 4 Collisional-nonequilibrium dissociation with $\tau_{p}=360 \mathrm{~ns}$ (see Figure 2).

Following characteristics of the reacting gas are shown in Figures 3-5: the temperature $T_{A B C}$ of excited $A B C$ molecules, the temperature $T_{A B}$ of resulting radicals $A B$ and the temperature $T_{b u f}$ of buffer gas molecules. Here we should note the following. The concept "temperature" has its usual sense only in the equilibrium case but, when the process is highly nonequilibrium, it is just a convenient parameter defining the average energy $E_{i}^{e q}$ of the truncated distribution function.

Besides the mentioned temperatures the time evolution of two other important values is plotted: the reaction yield $\beta$ of unimolecular dissociation (10a) and the so-called degree of nonequilibrium $\chi_{i}$ relating to the above-introduced values $E_{i}^{e q}, \bar{E}_{i}$ and $\delta_{i}$. It is defined as

$$
\chi_{i}=\frac{E_{i}^{e q}-\bar{E}_{i}}{\delta_{i}}
$$

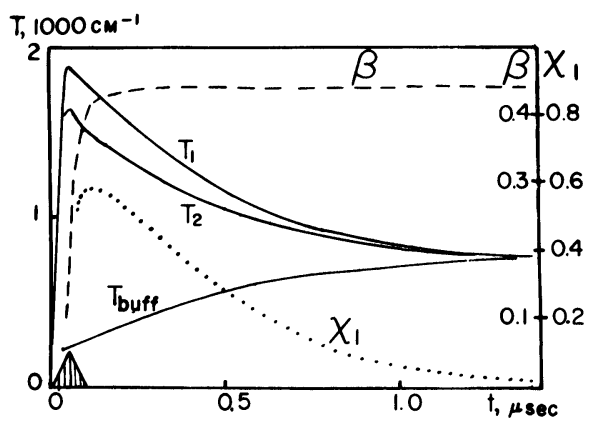

FIGURE 5 Collisionless dissociation with $\tau_{p}=30 \mathrm{~ns}$ (see Figure 2). 
The value $\chi_{i} \sim 1$ means that the distribution function is severely distorted by dissociation, i.e., the energy of truncation $E_{i}^{D}$ lies near the average thermal energy $E_{i}^{e q}$.

It may be seen from Figure 3 that at sufficiently long pulses $\left(\tau_{p}=\right.$ $5 \mu \mathrm{s})$ the reaction occurs under equilibrium conditions. Indeed, the temperatures of reagents $T_{A B C}, T_{A B}$ and that of buffer $T_{b u f}$ at all instants of time are practically equal. The dimensionless parameter of nonequilibrium of distribution function $\chi_{A B C}$ is always small (no higher than $7.3 \cdot 10^{-3}$ ). Generally speaking, whatever the pulse duration, any laser-induced reaction culminates (with $t>t_{e q}$ ) in such an equilibrium stage. But only in the case $\tau_{p} \geqslant t_{e q}$ it makes an essential contribution to the final reaction yield. The equilibrium reaction stage taking place in the time interval $\max \left(t_{e q}, \tau_{p}\right)<t<t_{\text {ext }}$ proceeds under self-cooling. ${ }^{35,41,42}$ Under these conditions the only source of cooling of the reacting mixture responsible for deceleration of the reaction is the reaction itself or rather the heat consumed by its elementary act (endoergicity). The total reaction yield $\beta$ and the temperature $T$ in this case turn out to be related by a simple equation expressing the law of conservation of energy. The dependence $\beta(T)$ proves to be nearly linear. ${ }^{42}$ For the reaction proceeding under self-cooling we can obtain analytical dependences of the yield on absorbed energy. They are investigated in detail in Ref. 35 .

Let us consider the reverse limiting case of very short pulses $\left(\tau_{p}=\right.$ $30 \mathrm{~ns}$, Figure 5) when the situation is qualitatively different. The reaction is basically near to completion when the buffer has not been substantially heated yet and its temperature is close to the initial one. In this sense the reaction with short $\tau_{p}$ can be termed collisionless. The degree of nonequilibrium also increases drastically and is as high as $\chi_{A B C} \simeq 0.6$.

Apart from a great difference between the temperatures of the both absorbing components $\left(T_{A B C}, T_{A B}\right)$ and the buffer temperature $T_{\text {buf, }}$, there is also a considerable difference between $T_{A B C}$ and $T_{A B}$, with $T_{A B C}$ always higher than $T_{A B}$. This means that at collisionless dissociation the product $A B$ is colder than the original substance $A B C$ which affects materially the kinetics of successive dissociation resulting in a qualitative change of product ratio (see Section 7). It is quite clear why the reaction products have a lower temperature $\left(T_{A B}<T_{A B C}\right)$. Some part of thermal energy of the original substance $A B C$ is inevi- 
tably spent to break the bond $A B-C$. So the vibrational energy and the temperature of $A B$ turn out to be considerably lower than those of $A B C$. Therefore the nonequilibrium initiation of $A B C$ dissociation causes the $A B$ ensemble to be cooled.

Finally, let us turn to Figure 4 where the course of reaction with $\tau_{p}=360 \mathrm{~ns}$ is is shown. The reaction basically proceeds under collisional-nonequilibrium conditions that is intermediate between collisionless and equilibrium ones. Indeed, if in the collisionless case the basic fraction of molecules dissociates before the buffer has time to be heated substantially $\left(T_{\text {buf }} \ll T_{A B C}\right)$ and, on the contrary, in the equilibrium case the main contribution to the dissociation is made by the instants of time when the buffer is already heated $\left(T_{\text {buf }} \simeq T_{A B C} \simeq\right.$ $T_{A B}$ ), the reaction illustrated in Figure 4 is intermediate between these two cases. The basic fraction of molecules here dissociates at the instants of time when the heating of the buffer comes about. In this sense the reaction is really nonstationary.

Another characteristic feature of collisional-nonequilibrium reaction can be found. if we consider behavior of absorbing molecules $A B C$, instead of the buffer gas. It can be seen from Figure 4, that after some initial transient its temperature $T_{A B C}$ is approximately "stabilized" at some quasistationary level. It is during this quasistationary phase of excitation the main part of reaction products is formed. The existence of such stabilization is related to mutual compensation for two opposite processes: the heating of $A B C$ gas by laser radiation and its cooling in the collisions with the buffer (and/or with colder dissociation products)

$$
\sigma_{A B C} I \simeq \nu n_{0}\left(T_{A B C}-T_{\text {buf }}\right)
$$

Here $\sigma_{A B C}$ is the absorption cross-section of $A B C, \nu n_{0}$ is the frequency of collisions between the $A B C$ molecule and the buffer. The value of $T_{A B C}$ and hence the reaction rate $d[A B C] / d t$ depend just on the instantaneous value of radiation intensity $I$ (rather than fluence $\Phi=$ $\left.\int_{-\infty}^{t} I\left(t_{1}\right) d t_{1}\right)$ and the temperature of different components. Such quasistationary dissociation does not arise either in collisionless or in equilibrium reactions because the basic fraction of molecules in these cases dissociates after the laser pulse is over. Some analytic expressions for dissociation rate for quasistationary reaction were derived in Ref. 15. 


\section{ROLE OF POSTPULSE DISSOCIATION}

Now we are going to consider the role of dissociation after the laser pulse is over. For this purpose, we calculate the value

$$
\eta_{p p}=\frac{[A B]\left(t_{\text {ext }}\right)-[A B]\left(2 \tau_{p}\right)}{[A B]\left(t_{\text {ext }}\right)}
$$

that makes a relative contribution to the final value $\beta=[A B]\left(t_{\mathrm{ext}}\right)$ of dissociation after the pulse action, i.e., when $2 \tau_{p}<t<t_{\text {ext }}$ (we must recall that the pulse shape is triangular and $2 \tau_{p}$ is its full length. The dependence $\eta_{p p}\left(\tau_{p}\right)$ is presented in Figure 6, the parameters of the model are the same as before. The external cooling time of reaction $t_{\mathrm{ext}}=100 \mu \mathrm{s}$. It may be seen that postpulse dissociation plays a decisive role $\left(\eta_{p p}\right.$ approximates 1$)$ in the limiting cases of short and long pulses. For short pulses this fact is well known from the works on "collisionless" MP dissociation and it is responsible for the existence of a great number of molecules excited over the dissociation limit but having no time to dissociate during a pulse. But, as the pulse duration increases, $\eta_{p p}$ begins to drop because the ratio $\tau_{p} / t_{c}$ decreases, i.e., a smaller and smaller fraction of molecules has time to dissociate during the time (of the order of $t_{c}$ ) when the collisions with the buffer have not brought about their deactivation yet.

With $\tau_{p}>t_{c}$ we turn to a collisional-nonequilibrium reaction when the dissociation of $A B C$ and the heating of the buffer occur simultaneously. The difference of their temperatures $T_{A B C}-T_{\text {buf }}$, responsible for the reaction rate, proves proportional to the radiation

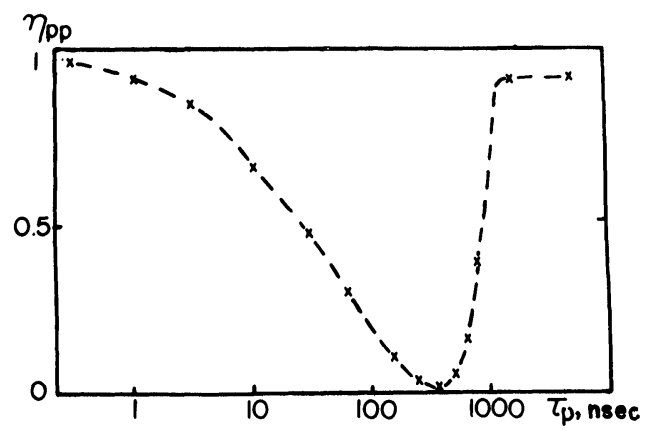

FIGURE 6 The dependence of the postpulse dissociation contribution $\eta_{p p}$ (Eq. (19)) on the pulse duration $\tau_{p}$, the total yield being constant $\beta=44 \%$. The parameters of the model are given in Table $I$. 
intensity. So the end of the laser pulse $(I=0)$ leads to a sharp stoppage of the reaction (see Figure 4), and so the reaction time for the collisional-nonequilibrium case approximates $2 \tau_{p}$. Therefore the postpulse dissociation contribution in this turns out to be extremely small reaching values less than one per cent.

With a further increase of $\tau_{p}$ with $\tau_{p} \geqslant t_{e q}$ the relaxation terminates during a pulse, and the whole reacting mixture can be described by one temperature $T$ (equilibrium conditions, see Figure 3 ). This temperature proves to be maximum by the completion of the pulse when the absorbed energy reaches its maximum. So only at the instant when the pulse is over the reaction rate acquires its maximum value. As a result, the dissociation during a pulse turns out to be unessential again and, with $\tau_{p}>t_{e q}, \eta_{p p}$ approximates unity.

So we have shown that, according to the relation between the laser pulse duration and two collisional times, the reaction may proceed both during a pulse (collisional-nonequilibrium reaction, $t_{c}<\tau_{p}<t_{e q}$ ) and after the pulse is over (collisionless and equilibrium reactions, $\tau_{p} \ll t_{c}$ and $\tau_{p}>t_{e q}$ respectively).

\section{POSSIBILITY OF SYNTHESIS OF LABILE INTERMEDIATE PRODUCTS}

In this section we shall take into account the possibility of participation of primary dissociation products in further reactions. Let us consider the case when the $A B$ product not only absorbs laser radiation but also can dissociate according to (1b). The kinetics of such successive dissociation depends on the relation between the rates of primary and secondary dissociation and, first of all, on their corresponding bond energies $D_{A B C}$ and $D_{A B}$. When the second dissociation is slower $\left(D_{A B C}<D_{A B}\right)$, we really deal with successive dissociation when with an increase in absorbed energy first we can observe the first dissociation (1a) and, only as the yield approximates $100 \%$, the second dissociation (1b) is turned on. So, in fact, two independent simple dissociations considered in the foregoing sections take place since reactions (1a) and (1b) proceed in different regions of absorbed energy and in the both cases the initial concentration of original substances $(A B C$ and $A B$ respectively) is equal or close to $100 \%$. All the said holds true for all regimes of dissociation considered and so this case does not need a special consideration. 
The situation is qualitatively different in the reverse case when the intermediate substance $A B$ is labile and dissociates faster than it is formed $\left(D_{A B C}>D_{A B}\right.$ and $\left.k_{A B C}^{U M} \ll k_{A B}^{U M}\right)$. At thermal initiation of reaction or under pulsed equilibrium conditions the temperatures of all the components are the same (for example, $T_{A B C}=T_{A B}$ ) and so the condition $k_{A B C}^{U M} \ll k_{A B}^{U M}$ means that it is impossible to obtain considerable concentrations of $A B$. Yet the reactions under collisionless and collisional-nonequilibrium conditions proceed in the absence of equilibrium $\left(T_{A B}<T_{A B C}\right.$, see Section 5) which opens up possibilities for effective synthesis of labile $A B$ substance. ${ }^{7,23,34,35,43-46}$ The study of this characteristic feature will be the subject of the present section. This new method of synthesis of labile substances can find wide practical application. One of the first step in this direction was the demonstration of the possibility of a high coefficient of conversion of $\left(\mathrm{CF}_{3}\right)_{3} \mathrm{CBr}$ to $\left(\mathrm{CF}_{3}\right)_{3} \mathrm{CI}$ at nonequilibrium IR laser dissociation ${ }^{34}$ which is impossible at thermal heating due to the radical $\left(\mathrm{CF}_{3}\right)_{3} \mathrm{C}$ being labile. With such applications in mind the $A B$ substance will be called below "desired" and its yield $n_{A B}\left(t_{\text {ext }}\right)=\beta_{d}$ the desired product yield.

Figure 7 presents the dependence of the total reaction yield $\beta=$ $1-n_{A B C}=n_{A B}+n_{A}$ and the relative desired product yield $\beta_{d} / \beta=$ $[A B] /([A B]+[A])$ characterizing reaction directivity on absorbed energy $\varepsilon$. With small $\varepsilon$ and $\beta \ll 1$ the value $\beta_{d} / \beta$ always tends to unity. Indeed, no matter how great the second dissociation rate may be, it is always possible to choose such a small value of $\beta$ that $\beta \approx n_{A B}$ and $n_{A}<n_{A B}$ (since $n_{A B} \propto(\Delta t)^{2}$ ). As the absorbed energy (and the yield $\beta$ ) is increased, the value of $\beta_{d} / \beta$ drops. Figure 7 agrees qualitatively with the results ${ }^{34}$ on synthesis of $\left(\mathrm{CF}_{3}\right)_{3} \mathrm{CI}$.

The dependence of $\beta_{d} / \beta$ on $\beta$ is of interest for analyzing the possibility of realizing high-directivity chemical synthesis. For highly effective synthesis of desired product it is necessary that the high values of $\beta_{d} / \beta$ characteristic of low yields should be kept up at sufficiently high values of $\beta$, too. In other words, the conditions $\beta_{d} / \beta \sim 1$ and $\beta \sim 1$ should be met simultaneously. As may be seen from Figure 8 , they are incompatible for a thermal reaction under stationary conditions: the dashed line plotted for this case for $T=$ $1500 \mathrm{~K}$ lies in the region of $\beta_{d} / \beta \ll 1$ with $\beta \sim 1$. Figure 8 also presents (solid lines) the dependence of $\beta_{d} / \beta$ on $\beta$ for reactions initiated by pulsed IR laser radiation at varying pulse durations (the value of $\tau_{p}$ is given near the curves). 


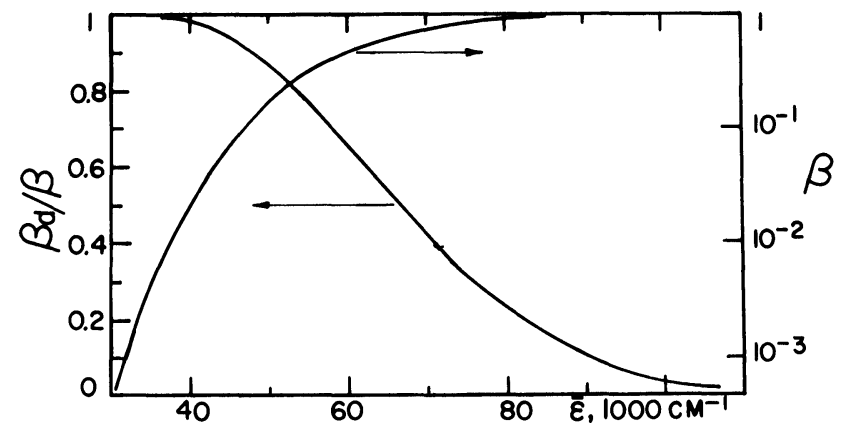

FIGURE 7 The reaction yield $\beta$ and directivity $\beta_{d} / \beta$ as a function of absorbed energy $\varepsilon$. The parameters of the system correspond to those given in Table I except for $\tau_{p}=100 \mathrm{~ns}, t_{\mathrm{ext}}=10 \mu \mathrm{s}$.

The reaction directivity $\beta_{d} / \beta$ is a minimum for an equilibrium reaction $\left(\tau_{p}=1.5 \mu \mathrm{s}\right)$. It should be noted however that even in this worst case $\beta_{d} / \boldsymbol{\beta}$ for pulsed (nonstationary) synthesis turns out to be greater than for a stationary reaction (dashed line). This is due to the fact that it is easy to attain high temperatures in nonstationary reactions (an increase in temperature decreases the thermal ratio $k_{A B}^{U M} / k_{A B C}^{U M}$ of reaction rates. As $\tau_{p}$ decreases, the curves $\beta_{d} / \beta(\beta)$ move upwards;

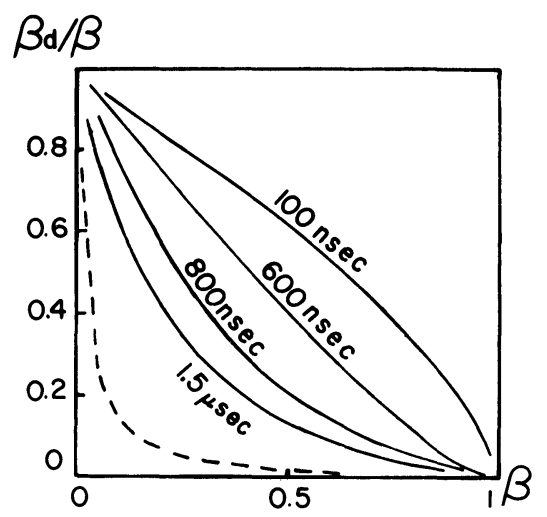

FIGURE 8 The dependence of the synthesis directivity $\beta_{d} / \beta$ on the total yield $\beta$ at different pulse durations $\tau_{p}$ (given near the curves), $t_{\mathrm{ext}}=10 \mu \mathrm{s}$. The rest of the parameters are given in Table $I$. The dashed line is the stationary thermal reaction calculated with the same parameters and $T=1500 \mathrm{~K}$. 
this shift is rather small while the equilibrium exists but in the transient region to collisional-nonequilibrium conditions the increase of $\beta_{d} / \beta$ is the most rapid (see the curves with $\tau_{p}=0.6 \mu \mathrm{s}$ and $0.8 \mu \mathrm{s}$ ). With a further decrease of $\tau_{p}$ under the collisional-nonequilibrium regime the increase of $\beta_{d} / \beta$ is decelerated again and, as the collisionless condition is established, it almost disappears. Thus, $\tau_{p}=0.1 \mu \mathrm{s}$ provides the maximum possible directivity of reaction. The curve with $\tau_{p}=0.1 \mu \mathrm{s}$ demonstrates the possibility of obtaining high directivities $\beta_{d} / \beta$ at high yields $\beta$ in pulsed nonequilibrium reactions. As $\beta$ increases, the value of $\beta_{d} / \beta$ drops rather slowly and with the $50 \%$ yield the directivity comes to $62 \%$. The value of $\beta$ being the same, the directivity at pulsed equilibrium dissociation equals just $12 \%$ and drops to $1.5 \%$ in the case of stationary thermal synthesis.

It should be stressed that due to a high rate of secondary dissociation at thermal equilibrium $\left(T_{A B}=T_{A B C}\right)$ we would always have $\beta_{d} / \beta \ll 1$. With our choice of parameters it is impossible to achieve $\beta_{d} / \beta \sim 1$ (at high values of $\beta$ ) at any temperatures since we believe that the second dissociation (1b) has not only a lower activation energy $\left(D_{A B C}=24000 \mathrm{~cm}^{-1}, D_{A B}=22000 \mathrm{~cm}^{-1}\right)$ but also a higher preexponential factor $\left(A_{A B}=10 \cdot A_{A B C}\right)$. Thus, for the rate ratio of reactions (1b) and (1a) we have: $k_{A B}^{U M} / k_{A B C}^{U M}=1000$ with $T=434 \mathrm{~cm}^{-1}(625 \mathrm{~K})$, $k_{A B}^{U M} / k_{A B C}^{U M}=100$ with $T=868 \mathrm{~cm}^{-1}(1250 \mathrm{~K})$ and $k_{A B}^{U M} / k_{A B C}^{U M}>10$ at any $T$.

It should be noted also, that we considered the case, when laser radiation absorption cross-section is the same for $A B$ and $A B C$. But the absorption band of $A B$ may not coincide with the band of $A B C$, and so in many cases $\sigma_{A B}<\sigma_{A B C}$. This will lead to a further decrease of the temperature of $A B$ (in comparison with $T_{A B C}$ ) and an appropriate drop of its dissociation rate which, in its turn, bring about a further increase of reaction directivity $\beta_{d} / \beta$. In this sense the values presented should be considered as lower bounds for $\beta_{d} / \beta$ in a laser-induced reaction.

\section{EFFECT OF BUfFER GAS PRESSURE}

Figure 9 presents the dependences of $\beta$ and $\beta_{d} / \beta$ in the reaction of successive dissociation $(1 \mathrm{a}, \mathrm{b})$ on the buffer gas pressure $P_{\text {buf }}$ with $\varepsilon=80000 \mathrm{~cm}^{-1} ; \tau_{p}=100 \mathrm{~ns}$ and $t_{\mathrm{ext}}=10 \mu \mathrm{s}$. The rest of the parameters 


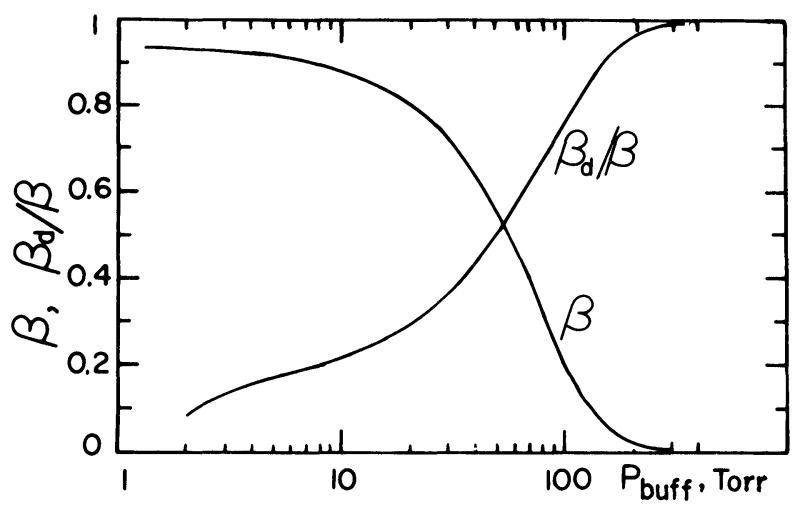

FIGURE 9 The dependence of the reaction yield $\beta$ and directivity $\beta_{d} / \beta$ on the buffer pressure $P_{\text {buf }}$ with $\varepsilon=80000 \mathrm{~cm}^{-1}, \tau_{p}=100 \mathrm{~ns}, t_{\text {ext }}=10 \mu \mathrm{s}$. The rest of the parameters are given in Table $\mathrm{I}$.

of the irradiated mixture are the same as before (see Table I). As $P_{\text {buf }}$ increases, this increases deactivation and decreases $T_{A B C}$ and $T_{A B}$ which causes a drastic fall of the total yield $\beta$. As a result of this decrease in $\beta$ (see Figure 8 ), the reaction directivity $\beta_{d} / \beta$ rises. Such dependences were observed in Ref. 34 where $I_{2}$ acted as a buffer gas.

To clear up the effect of buffer pressure on the reaction directivity related to the change of reaction conditions but not conditioned by the variation of the total yield $\beta$ we have carried out a special series of calculations (Figure 10) in which, along with $P_{\text {buf }}$, the absorbed energy $\varepsilon$ varies, too, so that the reaction yield remained constant $(\beta=45 \%)$. The value $P_{\text {buf }}$ includes, apart from the true pressure of buffer gas, "an internal buffer", i.e., translational and rotational degrees of freedom of active molecules. It can be seen that both too high and too low pressures are undesirable from the standpoint of obtaining maximum reaction directivity. The collisional-nonequilibrium reaction, when $t_{c}<\tau_{p}<t_{e q}$, is optimal in this case.

The decrease of $\beta_{d} / \beta$, as the buffer pressure drops, is explained by the fact that the role of the postpulse equilibrium stage increases. As it follows from the results of Section 5 (Figure 3), after the same temperature is set up in the reacting mixture, it proves to be still high enough for the final reaction yield to be determined by this equilibrium stage. But under this condition due to the equality $T_{A B C}=T_{A B}$ the reaction directivity proves to be minimum (see Figure 8 ). As $P_{\text {buf }}$ is 


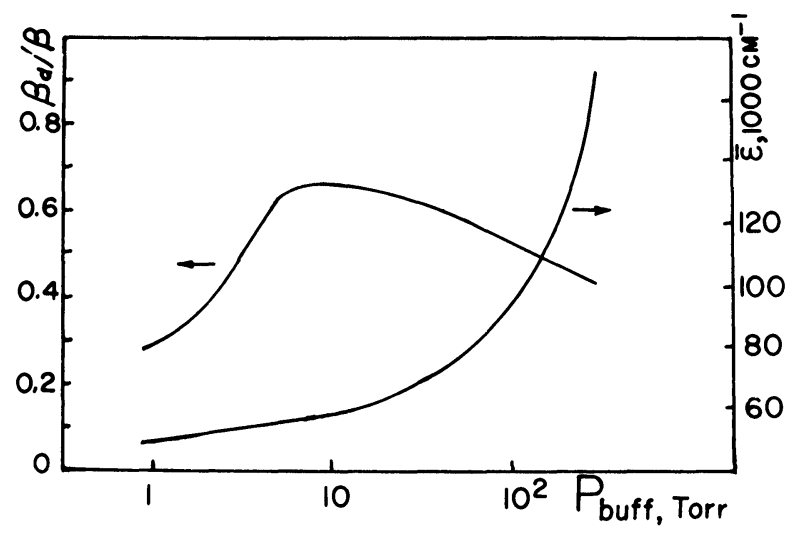

FIGURE 10 The dependence of the synthesis directivity $\beta_{d} / \beta$ on the buffer pressure $P_{\text {buf }}$ at the fixed yield $\beta=45 \%$. The absorbed energy $\varepsilon$ (right-hand scale) required to obtain this yield is also plotted. The rest of the parameters are the same as in Figure 8.

increased, the contribution of the equilibrium stage decreases and the reaction becomes collisional-nonequilibrium with a maximum difference $T_{A B C}-T_{A B}$ which suppresses the further dissociation of $A B$ and gives a maximum value of $\beta_{d} / \beta$.

A further increase of buffer pressure has a negative effect because the energy $\varepsilon$ necessary for obtaining the preset yield $(\beta=45 \%)$ begins to increase drastically and, besides, the very reaction directivity $\beta_{d} / \beta$ is somewhat reduced. So we can see that an optimal buffer pressure exists that enables us to obtain the maximum reaction directivity. It can be realized in the case when the buffer heat capacity is approximately equal to the heat capacity of excited molecules.

\section{CONCLUSIONS}

In this paper a simple theoretical model has been developed for laser-induced chemical reactions proceeding under highly non-equilibrium conditions when the methods of calculation of conventional equilibrium chemical kinetics cannot be applied. We take into account not only the difference in "temperatures" of different mixture components but also the difference of vibrational distribution functions 
from the Boltzmann one by introducing their degree of nonequilibrium $\chi$. This parameter is very useful for describing the high-rate reactions under consideration which result in extremely high (up to 100\%) radical concentrations. Indeed, the value $\chi$ defines the degree of depletion (on account of dissociation) of the high-energy part of vibrational distribution function and so the degree of nonequilibrium $\chi$ determines the temperature of resulting products (radicals).

The approach described enables a qualitative description of the changes in the reaction product ratio, the reaction time and its other parameters in passing from equilibrium to highly non-equilibrium initiation that takes place when the laser pulse duration or the gas pressure decrease. It must be stressed that such a simple approach cannot pretend to describe fully the system state. In particular, the presetting of the distribution with the use of only two parameters ( $T$ and $\chi$ ) is the strong approximation which cannot always provide a sufficient accuracy.

\section{References}

1. R. V. Ambartzumian and V. S. Letokhov, in: Chemical and Biochemical Applications of Lasers, Vol. 3, ed. C. B. Moore (Academic Press, New York, 1977), p. 167.

2. C. D. Cantrell and J. L. Lyman, in: Laser Handbook, Vol. 3, ed. M. L. Stitch (North-Holland, Amsterdam, 1979), p. 485.

3. P. A. Schulz, Aa. S. Sudbo, D. J. Krajnovich, H. S. Kwok, Y. R. Shen and Y. T. Lee, Ann. Rev. Phys. Chem. 30, 379 (1979).

4. V. N. Bagratashvili, V. S. Letokhov, A. A. Makarov and E. A. Ryabov, Laser Chem. 1,$211 ; 2,1$ (1983); 4, 171; 4, 311; 5, 53 (1984).

5. Laser Induced Chemical Processes, ed. J. I. Steinfeld (Plenum Press, New York, 1980).

6. Photoselective Chemistry, ed. J. Jortner, R. D. Levine and S. A. Rice (Wiley, New York, 1981), (Adv. Chem. Phys., Vol. 47).

7. V. N. Bagratashvili, M. V. Kuzmin and V. S. Letokhov, J. Phys. Chem. 88, 5780 (1984).

8. (a) M. F. Goodman, J. Stone and E. Thiele, in: Multiple Photon Excitation and Dissociation of Polyatomic Molecules, ed. C. D. Cantrell (in press); (b) V. S. Letokhov and A. A. Makarov, Uspekhi Fiz. Nauk (Russian) 134, 45 (1980); (c) H. W. Galbraith and J. R. Ackerhalt, in: Ref. 5, p. 1; (d) M. Quack, Adv. Chem. Phys. 50, 395 (1982).

9. See, for example, recent papers: (a) J. C. Stephenson, S. E. Bialkovski, D. S. King, E. Thiele, J. Stone and M. F. Goodman, J. Chem. Phys. 74, 3905 (1981); (b) V. M. Akulin, N. V. Karlov and A. M. Prokhorov, Kvantovaya Elektron. (Russian) 9, 1955 (1982); (c) J. L. Buechele, E. Wetz and F. D. Lewis, J. Chem. Phys. 77, 3500 (1982); (d) L. I. Trakhtenberg and G. M. Milikh, Kvantovaya Elektron. (Russian) 9, 2537 (1982); (e) R. J. Weston, Jr. J. Phys. Chem. 86, 4864 (1982); (f) J. Barker, Chem. Phys. 77, 301 (1983); (g) H.-L. Dai, Chem. Phys. Lett. 96, 324 (1983); (h) J. S. Francisco, Z. Qingshi and J. I. Steinfeld, J. Chem. Phys. 78, 5339 (1983); (i) W. A. Jalenak and N. S. Nogar, J. Chem. Phys. 79, 816 (1983). 
10. (a) W. D. Lawrance, A. E. W. Knight, R. G. Gilbert and K. D. King Chem. Phys. 56, 343 (1981); (b) J. E. Eberhardt, R. B. Knott, A. W. Pryor and R. G. Gilbert, ibid., 69, 45 (1982); (c) J. E. Eberhardt, I. E. Hoare, D. A. Jonson, R. B. Knott, A. W. Prior and A. B. Wagh. ibid., 72, 41 (1982).

11. (a) D. C. Tardy and B. S. Rabinovitch, Chem. Rev. 77, 369 (1977); (b) J. Troe. J. Chem. Phys. 66, 4745 and 4758 (1977).

12. M. J. Rossi, J. R. Pladzievicz and J. R. Barker, J. Chem. Phys. 78, 6695 (1983).

13. H. Hippler, J. Troe and H. J. Wendelken, J. Chem. Phys. 78, 6709 (1983).

14. H. Hippler, J. Troe and H. J. Wendelken, J. Chem. Phys. 78, 6718 (1983).

15. M. V. Kuzmin and A. A. Stuchebrukhov, Chem. Phys. 83, 115 (1984).

16. D. Gutman, W. Braun and W. Tsang, J. Chem. Phys. 67, 4291 (1977).

17. A. V. Baklanov, Yu. N. Molin and A. K. Petrov, Chem. Phys. Lett. 68, 329 (1979).

18. R. B, Knott and A. W. Pryor, J. Chem. Phys. 71, 2946 (1979).

19. C. Reiser, F. M. Lussier, C. C. Jensen and J. I. Steinfeld, J. Am. Chem. Soc. 101, 350 (1979).

20. A. Ben-Shaul and Y. Haas, J. Chem. Phys. 73, 5107 (1980).

21. S. Koda, Y. Ohnuma and S. Tsuchiya, Bull. Chem. Soc. Japan 53, 3447 (1980).

22. J. S. Chou and E. R. Grant, J. Chem. Phys. 74, 384 (1981).

23. W. E. Farneth, M. W. Thomsen, N. L. Schultz and M. A. Davies, J. Am. Chem. Soc. 103, 4001 (1981).

24. I. Oref, J. Chem. Phys. 75, 131 (1981); ibid 77, 1253 (1982).

25. V. Starov, N. Selamoglu and C. Steel, J. Phys. Chem. 85, 320 (1981).

26. K. Takeuchu, O. Kurihara and R. Nakane, Chem. Phys. 54, 383 (1981).

27. J. C. Jang, D. W. Setser and W. C. Danen, J. Am. Chem. Soc. 104, 5440 (1982).

28. R. J. McCluskey and S. V. Babu, J. Phys. Chem. 86, 3210 (1982).

29. V. N. Sazonov, A. A. Stuchebrukhov and S. V. Zatsepin, Chem. Phys. 69, 459 (1982); V. N. Sazonov and I. E. Khromov, ibid. 76, 25 (1983).

30. F. Zhang, J. S. Francisco and J. I. Sheinfeld, J. Phys. Chem. 86, 2402 (1982).

31. X. Zheng, Z. Xu and F. Kong, Chem. Phys. Lett. 85, 418 (1982).

32. D. W. Setser, H. H. Nguyen and W. C. Danen, J. Phys. Chem. 87, 408 (1983).

33. V. T. Platonenko and N. A. Sukhareva, Kvantovaya Electron. (Russian) 10, 134 (1983); E. N. Martynova, V. T. Platonenko and N. A. Sukhareva, Khinicheskaya Physica (Russian) 1983, 1047.

34. (a) V. N. Bagratashvili, V. N. Burimov, L. E. Deev, V. S. Letokhov and A. P. Sviridov, Kvantovaya Electron. (Russian) 9, 423 (1982); (b) V. N. Bagratashvili, V. N. Burimov, M. V. Kuzmin, V. S. Letokhov and A. P. Sviridov, Laser Chem. 1, 133 (1983).

35. V. N. Bagratashvili, V. N. Burimov, M. V. Kuzmin and A. P. Sviridov, Technical Report 83-3, Research Center for Laser Technology, Acad. Sci. of the USSR, 1983.

36. M. V. Kuzmin, Chem. Phys. 81, 199 (1983).

37. M. Dilonardo, Nuovo Cim. 63B, 131 (1981).

38. P. G. Harper, R. G. Harrison, M. R. Humphries and P. John, Appl. Phys. B28, 185 (1982); P. John, M. P. Humphries, R. G. Harrison and P. G. Harper, J. Chem. Phys. 79, 1353 (1983); P. G. Harper, J. Phys. B: At. Mol. Phys. 16, 3787 (1983).

39. See, for example, C. Steel, V. Starov, R. Leo, P. John and R. G. Harrison, Chem. Phys. Lett. 62, 121 (1979); R. T. Bailey, F. R. Cruickhauk, D. Pugh, R. Guthrie, W. Johnstone, J. Mayer and K. Middleton, J. Chem. Phys. 77, 3453 (1982); H.-L. Dai, E. Specht, M. R. Berman and C. B. Moore, J. Chem. Phys. 77, 4494 (1982).

40. P. J. Robinson, K. A. Holbrook, Unimolecular Reactions (Wiley, New York, 1972).

41. N. N. Akinfiev, A. N. Oraevskii, A. V. Pankratov, S. E. Pankratov, V. P. Pimenov and A. N. Skachkov. Kvantovaya Electron. (Russian) 8, 1177 (1981). 
42. V. N. Bagratashvili, and M. V. Kuzmin, Khimicheskaya Physika (Russian) 3, 1081 (1984).

43. V. N. Bagratashvili, M. V. Kuzmin and V. S. Letokhov, Laser Chem. (1983) 4, 139 (1984).

44. C.-R. Mao, N. Presser, L.-S. John, R. M. Moriarty and R. J. Gordon, J. Am. Chem. Soc. 103, 2105 (1981).

45. H. H. Nguen and W. C. Danen, J. Am. Chem. Soc. 103, 6253 (1981).

46. F. D. Lewis, P. A. Teng and E. Weitz, J. Phys. Chem. 87, 1666 (1983). 